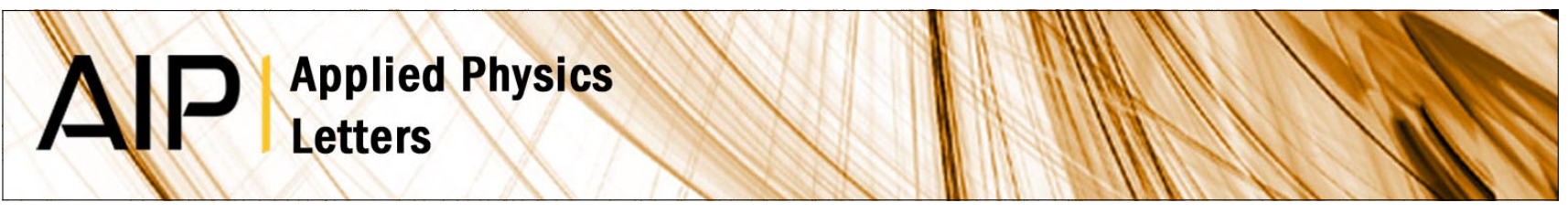

\title{
Photoresponse from noble metal nanoparticles-multi walled carbon nanotube composites
}

M. Scarselli, L. Camilli, L. Matthes, O. Pulci, P. Castrucci et al.

Citation: Appl. Phys. Lett. 101, 241113 (2012); doi: 10.1063/1.4771125

View online: http://dx.doi.org/10.1063/1.4771125

View Table of Contents: http://apl.aip.org/resource/1/APPLAB/v101/i24

Published by the American Institute of Physics.

\section{Related Articles}

On the alternatives for bath correlators and spectral densities from mixed quantum-classical simulations J. Chem. Phys. 137, 224103 (2012)

Inhibition of plasmonically enhanced interdot energy transfer in quantum dot solids via photo-oxidation J. Appl. Phys. 112, 104302 (2012)

Highly efficient photochemical $\mathrm{HCOOH}$ production from $\mathrm{CO} 2$ and water using an inorganic system AIP Advances 2, 042160 (2012)

Photoisomerization for a model protonated Schiff base in solution: Sloped/peaked conical intersection perspective

J. Chem. Phys. 137, 22 A543 (2012)

Photochemistry in a dense manifold of electronic states: Photodissociation of $\mathrm{CH} 2 \mathrm{ClBr}$

J. Chem. Phys. 137, 22A539 (2012)

\section{Additional information on Appl. Phys. Lett.}

Journal Homepage: http://apl.aip.org/

Journal Information: http://apl.aip.org/about/about_the_journal

Top downloads: http://apl.aip.org/features/most_downloaded

Information for Authors: http://apl.aip.org/authors

\section{ADVERTISEMENT} \section{AIP $\begin{aligned} & \text { Applied Physics } \\ & \text { Letters }\end{aligned}$}

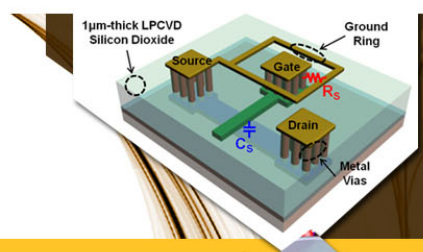

\section{SURFACES AND} INTERFACES

Focusing on physical, chemical, biological structural, optical, magnetic and electrical properties of surfaces and interfaces, and more..

\section{EXPLORE WHAT'S NEW IN APL}

SUBMIT YOUR PAPER NOW!
ENERCY CONVERSION AND STORACE 


\title{
Photoresponse from noble metal nanoparticles-multi walled carbon nanotube composites
}

\author{
M. Scarselli, ${ }^{1, a)}$ L. Camilli, ${ }^{1}$ L. Matthes, ${ }^{1,2}$ O. Pulci, ${ }^{1,3}$ P. Castrucci, ${ }^{1}$ E. Gatto, ${ }^{4}$ M. Venanzi, ${ }^{4}$ \\ and M. De Crescenzi ${ }^{1}$ \\ ${ }^{1}$ Dipartimento di Fisica, Università di Roma Tor Vergata, Via della Ricerca Scientifica 1, 00133 Roma, Italy \\ ${ }^{2}$ Institut für Festköpertheorie und optik, Friedrich Schiller Universität, Max-Wien Platz 1, Jena, Germany \\ ${ }^{3}$ ETSF, MIFO, and CNR-ISM, Via del Fosso del Cavaliere, Roma, Italy \\ ${ }^{4}$ Dipartimento di Scienze e Tecnologie Chimiche, Università di Roma Tor Vergata, Via della Ricerca \\ Scientifica 1, 00133 Roma, Italy
}

(Received 22 October 2012; accepted 27 November 2012; published online 12 December 2012)

\begin{abstract}
In this Letter, we investigated the photo-response of multi wall carbon nanotube-based composites obtained from in situ thermal evaporation of noble metals ( $\mathrm{Au}, \mathrm{Ag}$, and $\mathrm{Cu}$ ) on the nanotube films. The metal deposition process produced discrete nanoparticles on the nanotube outer walls. The nanoparticle-carbon nanotube films were characterized by photo-electrochemical measurements in a standard three electrode cell. The photocurrent from the decorated carbon nanotubes remarkably increased with respect to that of bare multiwall tubes. With the aid of first-principle calculations, these results are discussed in terms of metal nanoparticle-nanotube interactions and electronic charge transfer at the interface. (C) 2012 American Institute of Physics. [http://dx.doi.org/10.1063/1.4771125]
\end{abstract}

In the last two decades, carbon nanotubes (CNTs) have provided the scientific community with an excellent opportunity to find a fruitful match between experiment, theory, and simulation, as witnessed by the extensive literature. ${ }^{1,2}$ The CNTs exhibit so remarkable electronic and structural properties that they now constitute the building blocks in several nanoscale devices. ${ }^{3}$ Recently, the extended active surface area, good conductivity, and thermal stability of the CNTs have been exploited by interfacing them with a large variety of entities ranging from inorganic compounds, ${ }^{4}$ polymers, ${ }^{5}$ and biomolecules. ${ }^{6}$ These CNT-based hybrid systems exhibit new functions that originate from the cooperative effect of the distinctive properties of the two materials used, the CNTs and the nano-objects, and have found a broad range of applications. ${ }^{4-6}$ In this research field, an interesting class of derivatives can be obtained from the controlled deposition of metal and semiconductive compound nanoparticles (NPs) on the CNTs. ${ }^{4,7}$ In the case of metal NPs, the reported studies privileged transition metal NPs-CNT composites ( $\mathrm{Pt}, \mathrm{Pd}$, $\mathrm{Ru}, \mathrm{Ag}$, and $\mathrm{Au}$ ) since the majority of applications are in catalysis and gas sensing. ${ }^{9,10}$

Recently, our group investigated the morphology and composition of noble metals $(\mathrm{Ag}, \mathrm{Au}$, and $\mathrm{Cu}$ ) deposited on multi wall carbon nanotubes (MWCNTs) films via thermal evaporation and found that all three metals formed discrete nanoparticles of variable size on the tube outer wall. ${ }^{11}$

In this Letter, we report on the photo-electrochemical properties in the visible and near-ultraviolet (UV) photon energy range of these composites coating a $\mathrm{Si}(111)$ substrate used as a working electrode in a standard electrochemical cell. We demonstrate that all the three metals used $(\mathrm{Cu}, \mathrm{Au}$, and $\mathrm{Ag}$ ) enhance the photoresponse with respect to that of the bare MWCNTs, although each metal behaves differently depending on the NP average size. The electronic process that regulates the optical response of the composites is also

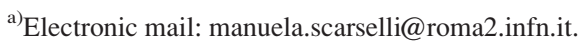

described theoretically, with the key parameter that dominates the observed increase being the charge transfer process at the interface from the metal NP to the CNT.

The synthesis of the metal NP-MWCNT composites was carried out into two subsequent steps: the growth of CNT film followed by in-situ metal deposition that generated the NPs. ${ }^{11}$

In brief, Fe $[1.00 \pm 0.01 \mathrm{~nm}$, nominal thickness (NT)] catalyst was thermally deposited on a cut piece of $\mathrm{Si}(111)$. The carbon nanotubes were grown by thermal chemical vapor deposition (CVD) using acetylene $\left(\mathrm{C}_{2} \mathrm{H}_{2}\right)$ gas atmosphere (200 sccm, 12 Torr) as carbon precursor while keeping the $\mathrm{Si}-\mathrm{Fe}$ sample at $750^{\circ} \mathrm{C}$, for $10 \mathrm{~min}$. The second step of the synthesis was the CNT films decoration. This task was performed in-situ under vacuum condition $\left(10^{-8}\right.$ Torr, base pressure) through a thermal evaporation process from a tungsten crucible, one for each metal, performed at the same deposition rate of $0.5 \AA / \mathrm{min}$. $\mathrm{Au}, \mathrm{Ag}$, and $\mathrm{Cu}$ were the metals used in the experiment. A set of three samples for each metal was synthesized simply by varying the evaporation time. The correspondent NT deposited was $0.20 \pm 0.01,0.50 \pm 0.01$, and $3.0 \pm 0.1 \mathrm{~nm}$, respectively. Field emission gun scanning electron microscopy (FEG-SEM, Leo Supra 35) was used to collect images directly on the MWCNT samples. Figures 1(a)1(c) report a collection of typical SEM images obtained on the MWCNT film after $3.0 \mathrm{~nm}$ NT deposition of Au (a), Ag (b), and $\mathrm{Cu}(\mathrm{c})$, respectively. From the images, it can be noticed that the metals deposition formed discrete NPs on the CNT outer wall with uniform dispersion, as expected from these metals. ${ }^{11,12}$ The MWCNT-based device photoresponse was measured with a conventional three-arm photo-electrochemical cell, using platinum (Pt) wire as the counter electrode and a saturated calomel electrode (SCE) as the reference electrode. A $0.5 \mathrm{M} \mathrm{KI}$ and $0.01 \mathrm{M} \mathrm{I}_{2}$ in acetonitrile solution was used as the electrolyte, the applied potential in all measurement being $12 \mathrm{mV}$, as shown in the scheme of Fig. 1(d). A $200 \mathrm{~W}$ Xe lamp (Osram) equipped with a monochromator was employed 

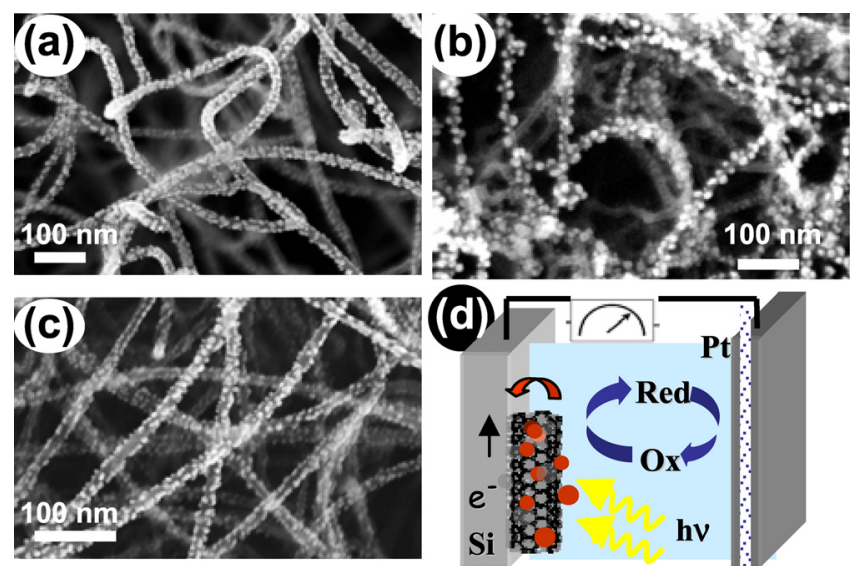

FIG. 1. SEM images of the MWCNT-based devices. (a) Typical image of the MWCNT film decorated with $\mathrm{Au}$ (a), Ag (b), and Cu (c) NPs, respectively. Images ((a)-(c)) have been obtained after thermal evaporation of $3 \mathrm{~nm}$ of metal NT. Scale bar is $100 \mathrm{~nm}$ in all images. (d) Schematic illustration showing the three electrodes cell set-up and the mechanism of the charge formation and collection upon light irradiation (solution $0.5 \mathrm{M} \mathrm{KI}$ and $0.01 \mathrm{M} \mathrm{I}_{2}$ in acetonitrile, $\mathrm{E}_{\text {cell }}=12 \mathrm{mV}$ ).

as the excitation source $(\lambda>300 \mathrm{~nm})$ and a PG-310 potentiostat (HEKA Elektronik, Lambrecht, Germany) was used for controlling the applied potential and photocurrent intensity measurements. The intensity of the light at the excitation wavelength near the electrode surface $\left(50 \mathrm{~mW} / \mathrm{cm}^{2}\right)$ was determined by azobenzene actinometry. ${ }^{13}$ The photoresponse of the samples was evaluated in terms of incident photon-to-charge carrier generation efficiency (IPCE) ${ }^{14}$ directly from the time traces of the on-off irradiation cycles, like those reported in Figure 2.

Ab-initio simulations were performed within density functional theory (DFT) in the local density approximation $(\mathrm{LDA})^{15}$ using the ESPRESSO package. ${ }^{16}$

Thanks to the large diameter of the CNTs, the curvature could be safely neglected and thus the entire hybrid system was simulated using a graphene layer with a metal nanoparticle on top. Due to computational limitations, the basic structural and electronic modifications of the graphene when decorated with $\mathrm{Cu}, \mathrm{Ag}$, and $\mathrm{Au}$ metal clusters have been computed for NP diameters ranging from 0 (1 single metal atom) to $\sim 0.8 \mathrm{~nm}$ (38 atoms) metal clusters. A graphene $9 \times 9 \times 1$ supercell ( $162 \mathrm{C}$ atoms) has been used for the largest NP. The geometry of the composite system was relaxed till the forces were less than $10^{-3}$ a.u. A set of up to $2 \times 2 \times 1 \mathrm{k}$-points was used to sample the Brillouin Zone, and we employed an energy cut-off of $64 \mathrm{Ry}$. Smaller clusters were studied with $4 \times 4 \times 1$ supercell ( $32 \mathrm{C}$ atoms) and, consequently, $9 \times 9 \times 1 \mathrm{k}$-points have been used in the Brillouin zone. Charge density analysis was performed within the Bader scheme. ${ }^{17}$

The application of interest of the CNT-based composites investigated focuses on their ability of producing a sizeable photoresponse in the visible and near ultraviolet energy range. To test such capability, the samples have been used as the electroactive layer coating the work electrode in a three electrode photo-electrochemical cell. A schematic of the measurement is reported in Figure 1(d). The photo-generated charges are collected at the Si surface and transported to the counter electrode via the external circuit. The red-ox couple $\mathrm{I}_{3}{ }^{-} / \mathrm{I}_{2}$ in solution sustains the electron flow through the cell

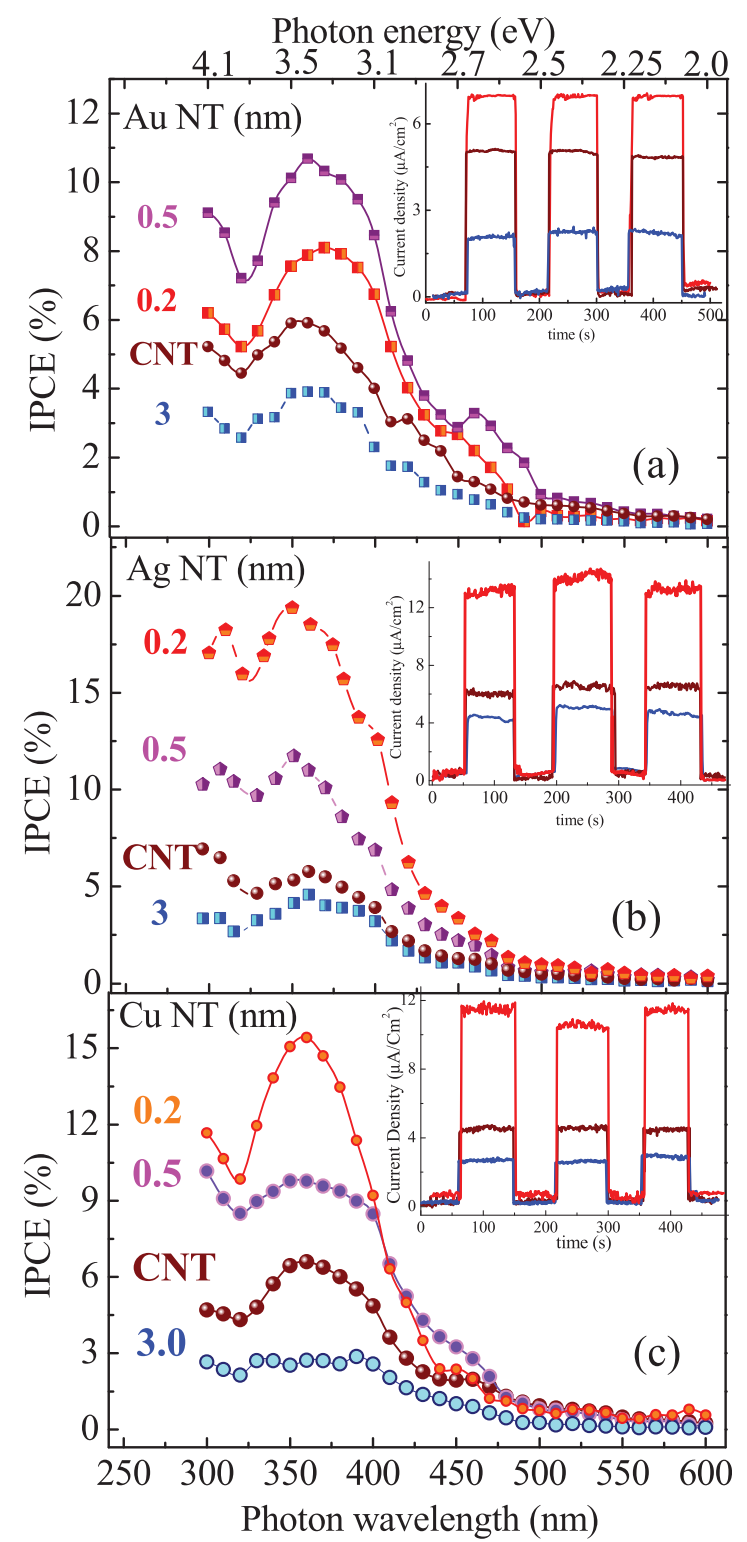

FIG. 2. (a)-(c) Evaluated results of the IPCE from the three MWCNThybrids and the bare MWCNT film (brown line-symbol) as a function of the incident photon wavelength. (a) Au-MWCNT, (b) Ag-MWCNT, and (c) CuMWCNT. The line-symbol colour corresponds to the same NT (orange for $0.2 \mathrm{~nm}$, violet for $0.5 \mathrm{~nm}$, and blue for $3 \mathrm{~nm} \mathrm{NT}$, respectively) used for all set of samples. The insets are three consecutive time traces in the $360-380 \mathrm{~nm}$ incident photon range of the on-off current density response acquired on the bare MWCNT film and two metal NP decorated-MWCNT samples (red line for $0.2 \mathrm{~nm}$, blue line $3 \mathrm{~nm} \mathrm{NT}$, and brown line for the MWCNT).

(Fig. 1(d)), giving rise to a steady anodic photocurrent, as shown in the insets of Fig. 2.

The photo-response from the three sets of samples is reported in terms of IPCE in Figures 2(a)-2(c) for each metal and nominal thickness used. The value can be compared to that acquired from the bare MWCNT film. Starting from Au-MWCNT samples of Figure 2(a), it can be noticed that for $0.2 \mathrm{~nm}$ and $0.5 \mathrm{~nm} \mathrm{NT}$, the signal is greater than that from MWCNTs, while that obtained from the $3 \mathrm{~nm}$ NT sample is lower in all photon spectral range studied. The maximum in the IPCE reaches $10.7 \%$ at around $370 \mathrm{~nm}$ for $0.5 \mathrm{~nm}$ NT sample compared to that of the MWCNTs at the same wavelength that is $5.9 \%$. Although the best photoresponse is concentrated around the ultraviolet energy range, it 
remains greater than that of the bare MWCNT film in all spectra. The maximum IPCE value from the bare MWCNT samples in Fig. 2 varied from $5.7 \%$ to $6.6 \%$. This can be ascribed to structural fluctuations in the CNT that influenced the optical response, as we have already reported. ${ }^{18}$

A similar trend in the IPCE curves has been obtained from the MWCNT samples decorated with $\mathrm{Ag}$ and $\mathrm{Cu}$, Figs. 2(b) and 3(c), respectively. Again the observed trend indicates that a moderate deposition of metal on the tube walls increases the photoresponse. In the case of $\mathrm{Ag}$, the signal grows up to $19.4 \%$ around $370 \mathrm{~nm}$, that is three times higher than that of the CNT that is only 5.7\%. No appreciable effect was observed for $3 \mathrm{~nm}$ NT. Finally, Figure 3(c) collects the IPCE from the Cu-MWCNT samples. The IPCE evaluated from the $\mathrm{Cu}-\mathrm{MWCNT}$ film reaches a maximum of $15.4 \%$ at $360 \mathrm{~nm}$ compared to that of the MWCNT of $6.6 \%$ for $0.2 \mathrm{~nm}$ NT. The maximum IPCE value is located in the ultraviolet spectral region, confirming the results reported in similar recent experiments. ${ }^{18-20}$ The maximum IPCE values from the three sets of samples and that of the MWCNT have been collected in Table I for better clarity.

The insets in Figs. 2(a)-2(c) show three consecutive time traces in the $360-380 \mathrm{~nm}$ incident photon range of the on-off current density response acquired on the bare

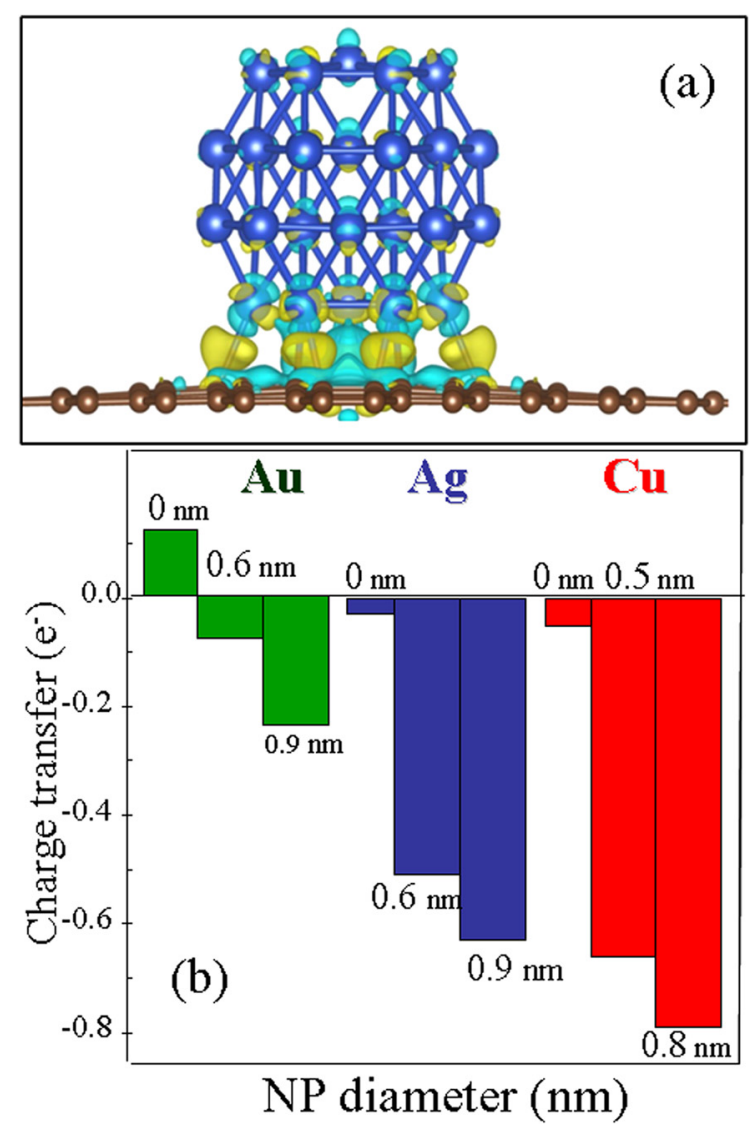

FIG. 3. DFT calculations for a simplified CNT+NP hybrid system, here, modelled by metal clusters on top of a graphene layer. (a) Simulation of a $\mathrm{Cu} \mathrm{NP}(\mathrm{d}=0.8 \mathrm{~nm})$ on top of a graphene layer. Charge depletion areas are shown in light blue and charge accumulation areas in yellow. (b) Charge transfer from the NP to the graphene substrate $($ when $<0)$ and vice-versa $($ when $>0$ ). The charge transfer from the metal to the graphene substrate increases with increasing NP diameter. 0 diameter NP refers to 1 atom cluster.
TABLE I. IPCE maximum values obtained metal NP-MWCNT films and the reference MWCNT film, from the curves reported in Figs. 2(b)-2(d).

\begin{tabular}{lrrrrrr}
\hline \hline $\mathrm{NT}^{\mathrm{a}}$ & $\mathrm{Au}^{\mathrm{b}}$ & $\%^{\mathrm{d}}$ & $\mathrm{Ag}^{\mathrm{b}}$ & $\%^{\mathrm{d}}$ & $\mathrm{Cu}^{\mathrm{b}}$ & $\%^{\mathrm{d}}$ \\
\hline 0.2 & 8.0 & 36 & 19.4 & 240 & 15.4 & 133 \\
0.5 & 10.7 & 81 & 11.7 & 105 & 9.8 & 46 \\
3 & 3.9 & -34 & 4.6 & -20 & 2.7 & -60 \\
$\mathrm{MWCNT}^{\mathrm{c}}$ & 5.9 & $\ldots$ & 5.7 & & 6.6 & $\ldots$ \\
\hline \hline
\end{tabular}

${ }^{\mathrm{a}} \mathrm{NT}$ is the metal nominal thickness (nm).

${ }^{\mathrm{b}}$ Maximum IPCE value (\%) found for each metal.

${ }^{\mathrm{c}}$ Maximum IPCE value (\%) found for the MWCNT reference sample.

${ }^{\mathrm{d}}$ Relative increment or decrement of the IPCE (\%) for each metal with respect to the bare MWCNT at the maximum.

MWCNT film and two metal NP decorated-MWCNT samples $(0.2 \mathrm{~nm}$ and $3 \mathrm{~nm} \mathrm{NT})$. All samples showed a prompt response to incident light and the current signal is anodic in character. This means that the electrons flow from the metal NP/MWCNT composite to the collecting auxiliary electrode, as described in Fig. 1(d). It is important to underline that these measurements are very sensitive to the surface process, and the observed signal is produced and collected only at the metal MWCNT film-Si interface. In fact, photocurrent measurements performed on the Si substrate before and after the Fe catalyst deposition did not show any photocurrent response (data not shown). In addition, all the three metal NP-MWCNT composites studied exhibit a line shape that closely resembles that of the bare MWCNTs. This behaviour is different from that reported in case of similar studies on semiconductive NP-single wall carbon nanotube composites, where the response mainly mimics that of the NP with a maximum located around the NP absorption edge. ${ }^{21}$

The observed increase in the photocurrent signal that we obtained at the macroscopic level suggests the existence of a microscopic electronic mechanism enhancing the photocurrent efficiency. In particular, the observed trend (Figs. 2(a)2(c)) suggests that in the presence of metal NPs, additional charges are made available and efficiently collected at the electrode. This implies that a charge transfer might have occurred at the microscopic level in the entire hybrid system that involved both the CNT and the particles before light excitation. A charge transfer process for a metal single wall carbon nanotube (SWCNT) in close contact with a linear chain of $\mathrm{Cu}$ atoms was theoretically described by Kong et $a l^{22}$ The authors predicted an efficient electron transfer from the $\mathrm{Cu}$ atom in the chain located on top of the $\mathrm{C}$ atom in the tube. An increase in the density of states of the $\mathrm{C}$ atom was also found, thus, confirming that this simple hybrid system showed electronic properties remarkably different from those of the two starting constituents. In order to shed some light onto the microscopic mechanism that regulates the increased photocurrent, we have calculated, for graphene decorated with $\mathrm{Au}, \mathrm{Ag}$, and $\mathrm{Cu}$ NPs, the geometry and the electronic properties of the composite system for three different NPs size. Figure 3(a) reports the result of the simulation of a $\mathrm{Cu} N P(d=0.8 \mathrm{~nm})$ on top of a graphene layer where the charge depletion and accumulation areas have been determined. A similar trend was also found for $\mathrm{Au}$ and $\mathrm{Ag}$ and has not been reported. The charge transfer is mainly located at the interface between the NPs and the graphene 
layer, thus, confirming that the process involved in the photocurrent increase is mainly an interface-driven effect.

Moreover, the amount of charge transfer increases with the NP diameter starting from 1 atom cluster up to the largest one, as reported in Fig. 3(b). The $\mathrm{Ag}$ and $\mathrm{Cu}$ always show a similar performance, with a net electron transfer from the NP to the substrate at any size considered. Au simulation exhibits a slight different trend, being the charge transfer for the one-atom $\mathrm{Au}$ from graphene towards the metal NP, with an inversion for larger NPs. It must be noticed that at high metal coverage ( $3 \mathrm{~nm} \mathrm{NT})$, NPs increase in size and density so that they prevent light to reach CNT surface. In this case, the IPCE outcome is lower than that of bare CNTs and no appreciable effect could be detected.

Differences in Pauling electronegativity can qualitatively explain the different behaviour of $\mathrm{Au}, \mathrm{Ag}$, and $\mathrm{Cu}$ when put in contact with $\mathrm{C}$ substrate: $\mathrm{Ag}$ and $\mathrm{Cu}$ are less electronegative (1.9 Pauling electronegativity) than $\mathrm{C}(2.5)$, whereas that of Au electronegativity (2.4) is comparable with that of $\mathrm{C}$. Hence, charge-transfer from the NP towards the $\mathrm{C}$ substrate is more efficient for $\mathrm{Cu}$ and $\mathrm{Ag}$ NPs than for Au. It is noticeable that, given a fixed size of the NPs, the amount of charge transferred from the metal to the substrate is quantitatively very different (smaller) for $\mathrm{Au}$ with respect to $\mathrm{Ag}$ and $\mathrm{Cu}$. This is in agreement with the experimental results shown in Fig. 2(a) which shows just a moderate increase of photocurrent for $\mathrm{Au}$ when compared with $\mathrm{Cu}$ and $\mathrm{Ag}$.

In summary, we have demonstrated that MWCNT films decorated with noble metal NPs can be used to generate intense electric currents upon photon absorption in the visible and near ultraviolet region. Insights into the photogeneration mechanism can be inferred comparing the experimental results to the theoretical simulations. A mechanism of photo-induced charge generation at the interface is described in which the presence of the metal NPs on the tubes deeply modifies the electronic properties of the CNT and makes available additional charges that are subsequently involved in the photo generation process. The main consequence is that a significant increase in the photocurrent response of the NP/CNT composite is registered in all the photon spectral range explored.

M.S., L.C., P.C., and M.D.C. thank Professor F. Nanni from the University of Tor Vergata for the SEM measurements and acknowledge the financial support of the Air Force Office of Scientific Research Material Command, USAF, under Grant No. FA8655-11-1-306 and the Queensland Government smart futures fund National and Interna- tional Research Alliances Program (NIRAP) ABN 83791 724622 .

L.M. acknowledges financial support from EC project CLERMONT4 (Grant No. 235114).

This work was selected as an ETSF project (Project No. 448). We thank CINECA for granting CPU time.

${ }^{1}$ A. Jorio, M. S. Dresselhaus, and G. Dresselhaus, Carbon Nanotubes: Advanced Topics in the Synthesis, Structure, Properties, and Applications (Springer, New York, 2008).

${ }^{2}$ J.-C. Charlier, X. Blase, and S. Roche, Rev. Mod. Phys. 79, 677 (2007).

${ }^{3}$ R. H. Baughman, A. A. Zakhidov, and W. A. de Heer, Science 297, 787 (2002).

${ }^{4}$ V. Georgakilas, D. Gournis, V. Tzitzios, L. Pasquato, D. M. Guldi, and M. Prato, J. Mater. Chem. 17, 2679 (2007).

${ }^{5}$ M. Giulianini, E. R. Waclawik, J. M. Bell, M. Scarselli, P. Castrucci, M. De Crescenzi, and N. Motta, Appl. Phys. Lett. 95, 143116 (2009).

${ }^{6}$ X. Guo, L. Huang, S. O’Brien, P. Kim, and C. Nuckolls, J. Am. Chem. Soc. 127, 15045 (2005).

${ }^{7}$ V. M. Rotello, Nanoparticles: Building Blocks for Nanotechnology (Kluwer Academic/Plenum, New York, 2004).

${ }^{8}$ G. G. Wildgoose, C. E. Banks, and R. G. Compton, Small 2, 182 (2006).

${ }^{9}$ A. Goldoni, L. Petaccia, S. Lizzit, and R. Larciprete, J. Phys. Condens. Matter 22, 013001 (2010).

${ }^{10}$ Z. Zanolli, R. Leghrib, A. Felten, J.-J. Pireaux, E. Llobet, and J.-C. Charlier, ACS Nano 5, 4592 (2011).

${ }^{11}$ M. Scarselli, L. Camilli, P. Castrucci, F. Nanni, S. Del Gobbo, E. Gautron, S. Lefrant, and M. De Crescenzi, Carbon 50, 875 (2012).

${ }^{12}$ Y. Zhang, N. W. Franklin, R. J. Chen, and H. Dai, Chem. Phys. Lett. 331, 35 (2000).

${ }^{13}$ H. J. Kuhn, S. E. Braslavsky, and R. Schmidt, Pure Appl. Chem. 61, 187 (1989).

${ }^{14}$ The IPCE (incident photon-to-current efficiency) was determined using the equation: $\operatorname{IPCE}(\%)=\frac{\mathrm{i}\left(\mathrm{Acm}^{-2}\right) \times 1240}{\mathrm{I}\left(\mathrm{W} \mathrm{cm}^{-2}\right) \times \lambda(\mathrm{nm})} \times 100$ where $\mathrm{i}$ is the short circuit photocurrent $\left(\mathrm{A} / \mathrm{cm}^{2}\right), \mathrm{I}$ is the incident light intensity $\left(\mathrm{W} / \mathrm{cm}^{2}\right)$, and $\lambda$ is the incident photon wavelength (nm). For details see A. C. Khazraji, S. Hotchandani, S. Das, and P. V. Kamat, J. Phys. Chem. B 103, 4693 (1999).

${ }^{15}$ W. Kohn and L. J. Sham, Phys. Rev. 140, A1133 (1965); P. Hohenberg and W. Kohn, ibid. 136, B864 (1964); D. M. Ceperley and B. J. Alder, Phys. Rev. Lett. 45, 566 (1980); J. P. Perdew and A. Zunger, Phys. Rev. B 23, 5048 (1981).

${ }^{16}$ P. Giannozzi, S. Baroni, N. Bonini, M. Calandra, R. Car, C. Cavazzoni, D. Ceresoli, G. L. Chiarotti, M. Cococcioni, I. Dabo et al., J. Phys.:Condens. Matter 21, 395502 (2009).

${ }^{17}$ G. Henkelman, A. Arnaldsson, and H. Jónsson, Comput. Mater. Sci. 36, 354 (2006).

${ }^{18}$ P. Castrucci, F. Tombolini, M. Scarselli, E. Speiser, S. Del Gobbo, W. Richter, M. De Crescenzi, M. Diociaiuti, E. Gatto, and M. Venanzi, Appl. Phys. Lett. 89, 253107 (2006).

${ }^{19}$ M. Scarselli, C. Scilletta, F. Tombolini, P. Castrucci, M. Diociaiuti, S. Casciardi, F. Tombolini, E. Gatto, M. Venanzi, and M. De Crescenzi, J. Phys. Chem. C 113, 5860 (2009).

${ }^{20}$ M. Scarselli, P. Castrucci, L. Camilli, S. Del Gobbo, S. Casciardi, F. Tombolini, E. Gatto, M. Venanzi, and M. De Crescenzi, Nanotechnology 22, 035701 (2011).

${ }^{21}$ I. Robel, B. A. Bunker, and P. V. Kamat, Adv. Mater. 17, 2458 (2005).

${ }^{22}$ K. Kong, S. Han, and J. Ihm, Phys. Rev. B 60, 6074 (1999). 